\title{
The AMEDEE nuclear structure database
}

\author{
S. Hilaire ${ }^{\mathrm{a}}$ and M. Girod \\ CEA/DAM Île-de-France, DPTA/Service de Physique Nucléaire, BP. 12, 91680 Bruyères-le-Châtel, France
}

\begin{abstract}
The increasing need for nuclear data far from the valley of stability requires information on nuclei which cannot be accessed experimentally or for which almost no experimental data is known. Consequently, the use of microscopic approaches to predict properties of such poorly known nuclei is necessary as a first step to improve the quality of nuclear data evaluations. Within this context, large scale mean field calculations from proton to neutron drip-lines have been performed using the Hartree-Fock-Bogoliubov method based on the D1S Gogny nucleon-nucleon effective interaction. Nearly 7000 nuclei have been studied under the axial symmetry hypothesis and several properties are now available for the nuclear scientific community on an Internet web site for every individual nucleus. Some global properties will be presented, such as the positions of the drip-lines, the nuclide ground state deformations and binding energies as well as regions where possible super or hyper-deformation might be encountered. The organization of the online database will also be described as well as its future extensions.
\end{abstract}

\section{Introduction}

The microscopic Hartree-Fock-Bogoliubov (HFB) method using the Gogny effective nucleon-nucleon interaction D1S $[1,2]$ has shown for more than 20 years its predictive power in various aspects of nuclear structure [3-12]. However, it had never been applied from a systematic point of view to study nuclei from proton to neutron drip-lines. Thanks to the high computer power available nowadays, such a project has been recently fulfilled, (i) performing constrained axially symmetric $\mathrm{HFB}+\mathrm{D} 1 \mathrm{~S}$ calculations for nuclei from Carbon $(Z=6)$ up to Darmstadtium $(Z=110)$ for both even-even, odd mass and odd-odd nuclei and (ii) putting the obtained results online. The main advantage of this type of study is to use a coherent method with a unique set of parameters for all studied properties and all nuclei.

Similar projects have already been conducted using macroscopic-microscopic approaches [13,14], extended Thomas-Fermi with Strutinsky integral model [15] or other microscopic methods such as HFB method with Skyrme type interactions [16-18] or relativistic mean-field theory [19]. However, to our knowledge, all the obtained results are not available to the scientific community as a web site.

After a brief description of the content of the online database, we will discuss some global properties that can be extracted from our large scale approach, such as the prolate or oblate (or quasi-spherical) nature of the nuclear shapes or regions where nuclei may exhibit superdeformed or hyperdeformed bands. We will then discuss the deviation of the predicted binding energies from experimental data and also compare theoretical and experimental charge radii. We will finally mention future extensions of our database which will be of great interest to strengthen the connection of nuclear structure predictions with nuclear reaction calculations.

\footnotetext{
${ }^{a}$ Presenting author, e-mail: stephane.hilaire@cea.fr
}

\section{Content of the AMEDEE database}

Using the constrained axially symmetric HFB+D1S method, we have determined potential energy surfaces (PES) of nearly 7000 nuclei from proton to neutron drip line, as function of the axial deformation parameter $\beta$ defined by

$$
\beta=\frac{1}{A R^{2}} \sqrt{\frac{5 \pi}{9}} q_{20}
$$

where $R=1.2 A^{1 / 3}$ is the nucleus radius and $q_{20}$ is the quadrupole moment. In the present approach, our deformation parameter $\beta$ ranges between -0.6 and 1.3 in order to locate mass regions where nuclei might exhibit superdeformed as well as hyperdeformed states. For odd and odd-odd nuclei, the blocking technique without breaking time reversal symmetry has been used in order to determine also the spin of the nucleus ground state (GS). All the nuclear structure informations which have been obtained as functions of the $\beta$ parameter as well as technical details on the HFB+D1S method are displayed on an Internet web site whose address is http://www-phynu.cea.fr/HFB-Gogny.htm.

The nuclei considered in the database are those for which

$$
\begin{aligned}
& E_{H F B}(Z, N)<E_{H F B}(Z-2, N) \text { for the proton drip-line, } \\
& E_{H F B}(Z, N)<E_{H F B}(Z, N-2) \text { for the neutron drip-line. }
\end{aligned}
$$

Of course, we have tried other criteria to defined the drip-lines such as looking if the chemical potential remains negative or if the $Z^{\text {th }}$ or $N^{\text {th }}$ level is bound or not, but the differences are usually not significant and are certainly not worth a deep discussion in the present paper. It is worth mentioning that our drip-lines definition does not account for the fact that nuclei may fission instantaneously because of a too weak fission barrier.

On this web site, a large amount of information is displayed graphically or can be downloaded in a tabulated format for each nucleus as function of $\beta$. It is also possible to display a given isotopic or isotonic chain for any of the results available for a single nucleus. 


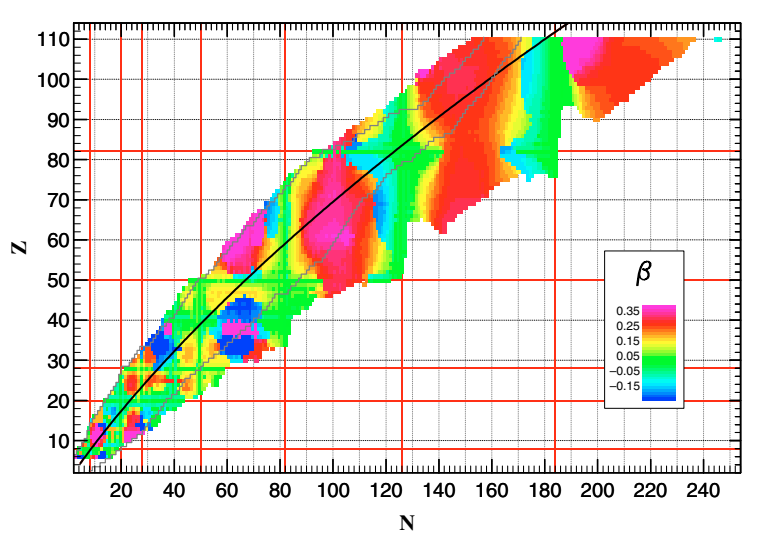

Fig. 1. Chart of the nuclides showing the deformation of the PES minima of all the nuclei contained in the database. The black line represents the stability line and the grey broken lines define the limits between which nuclear masses are experimentally known or extrapolated according to [20].

\section{Global qualitative properties}

For each nucleus of the AMEDEE database, we can locate the minimum of the PES which is the closest to sphericity $(\beta=0)$ and plot the chart of the nuclei as shown on figure 1. In most of the cases, this minimum is also the lowest point of the PES, and thus corresponds to the nucleus ground state. Only for very few nuclei is the lowest point of the PES not the closest to sphericity. However, such nuclei are very neutron rich and probably completly unstable when considering the fission process.

Several general observations can be made when looking at this chart. First, one can observe the clear appearance of nearly spherical mass regions (green zones) located around the well known magic numbers (red vertical and horizontal lines) 8 , $20,28,50,82,126$ as well as the one predicted for $N=184$. It is also worth noticing that for $N=82,126$ and 184 a clear "vertical wall" can be observed showing that these magic neutron numbers persist close to the neutron drip line. The same remark holds true for $Z=50$ and $Z=82$ close to the proton drip-line. Such a behavior cannot be observed for the other neutron/proton magic numbers. Between the spherical mass regions, well deformed nuclei $(\beta>0.2)$ are found among which the well known rare earths and actinides. Also, when the number of neutrons gets close to a magic number, a transition from a prolate $(\beta>0)$ to an oblate $(\beta<0)$ shape before getting spherical is clearly observed. This is particularly clear for $Z \approx 60-70$ where the neutron number crosses the $N=82$ as well as the $N=126$ magic shells. In fact when looking carefully at the PES of such nuclei, one can observe two minima which have almost the same energy. This feature characterizes either soft (and probably triaxial) nuclei or nuclei exhibiting shape coexistence.

When investigating deeper the PES contained in our database, we can also locate mass regions where the PES display other minima than that corresponding to the nucleus ground state. Such an analysis can be performed using several constrains, such as the depth of the secondary well, its deformation, or the difference $\Delta_{w}$ between the energy of the secondary well and that of the GS well. On figure 2, for instance, we have

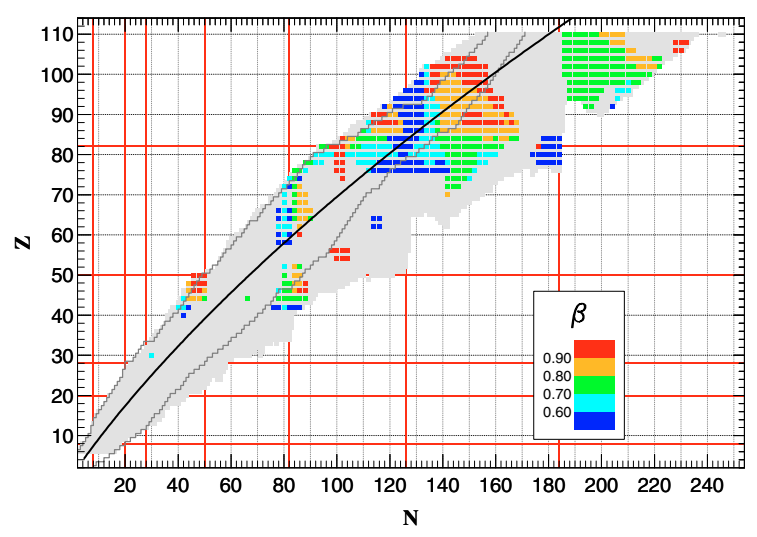

Fig. 2. Chart of the nuclides showing the possible mass regions where superdeformed states might show up with the corresponding deformations. The grey area corresponds to the nuclei studied in this paper. The black line represents the stability line and the grey broken lines define the limits between which nuclear masses are experimentally known or extrapolated [20].

considered all the nuclei for which (i) the secondary well depth is larger than $200 \mathrm{keV}$, (ii) the difference $\Delta_{w} \leq 30 \mathrm{MeV}$ and (iii) $0.5<\beta<1$. Similar studies had already been performed nearly 20 years ago $[11,12]$ to locate candidates for shape isomerism, using, however, more approximation than in the present case. If we compare our results with those of refs. [11,12], we find the same mass regions plus other regions rather located on the neutron rich side which had not been considered in refs. $[11,12]$. It is important to point out that the criteria we have used correspond to a severe situation with respect to rotational motion and one might find experimentally much more cases where superdeformed bands show up. Indeed, the $200 \mathrm{keV}$ criteria means that we do not include nuclei for which a minimum appears when rotational motion is accounted for, when no minimum is visible for the GS potential energy surface.

It is also important to mention that we are aware that a difference of $30 \mathrm{MeV}$ between the two well depth is quite high but one must not forget that the well depth alone does not give the true position of the levels. The curvature of the wells also plays a key role. Our apparently large $\Delta_{w}$ value thus permit to include nuclei for which the curvature of the wells is such that the energy difference between the levels located in these wells is much lower than $\Delta_{w}$.

\section{A few quantitative analysis}

Apart from the previous qualitative analysis, it is also interesting to compare a few nuclei ground state properties with available experimental data. Among the various properties that can be analysed, we have chosen to compare on figure 3 (resp. fig. 4) theoretical and experimental masses (resp. charge radii). On figure 3, the differences between theoretical and experimental masses are plotted as function of the neutron number $N$. Four types of nuclei are distinguished, namely odd-odd, odd-even, even-odd, as well as even-even nuclei. In each panel, the lines connect an isotopic chain. As can be seen, the global behavior of $\delta E$ does not depend on the odd-odd, odd or even-even nature of the nuclei, even if the calculations 


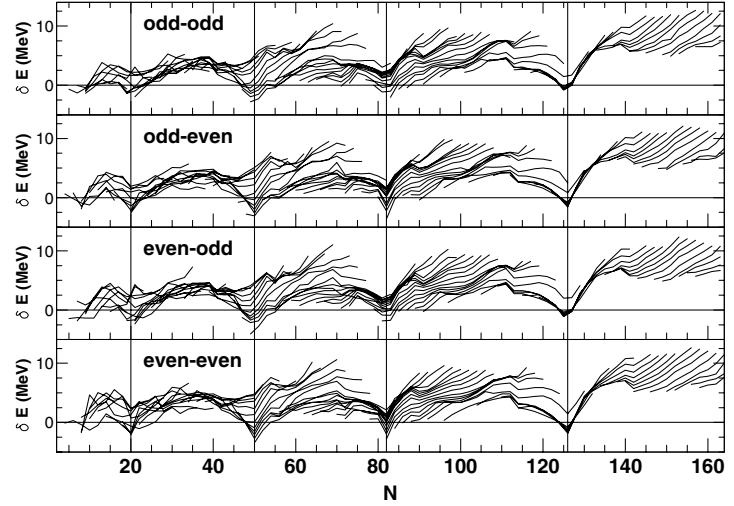

Fig. 3. Energy differences between theoretical and experimental masses of ref. [20] as functions of the neutron number.

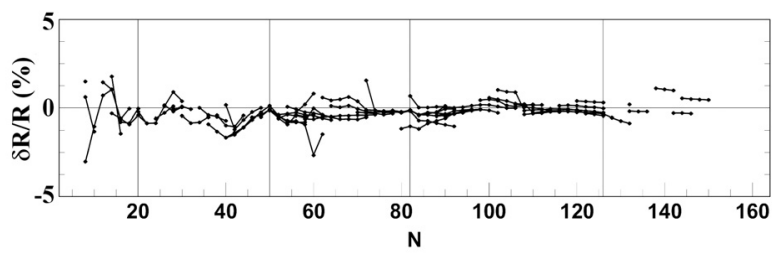

Fig. 4. Relative difference between theoretical (HFB axial) and experimental charge radii as function of the neutron number. Experimental radii are taken from ref. [22].

are more rigourous for even-even nuclei. Moreover, arches are clearly appearing with minima located around magic neutron numbers where nuclei are also overbound. However, we know that correlations beyond mean field will somehow wash out these arches. Finally, and more anoying is the increase of $\delta E$ with increasing neutron number $N$. This tendency, particularly clear for the heaviest nuclei, results from the fact that the neutron matter energy dependence of D1S has not been precisely investigated when the force was adjusted. This problem will be solved in a near future by re-adjusting the Gogny force parameters [21].

On figure 4, we compare the theoretical and experimental charge radii as functions of the neutron number. The agreement is quite satisfactory since the overall disagreement remains below roughly $2 \%$. However, a general trend is that the theoretical charge radii are systematically lower than the experimental values. Again, we known that correlations beyond mean field will have a significant impact on these comparisons.

\section{Future extensions of the database}

The very first extension of the database concerns a fundamental ingredient of semi-microscopic optical potentiel calculation: the nuclear matter densities. As shown in ref. [23], and illustrated on figure 5 for the present discussion, fairly good optical model predicitions can be obtained when constructing optical potentials out of HFB + D1S radial matter densities. For this reason, the next update of the AMEDEE database will contain, on top of all the results already available, the required nuclear matter densities.

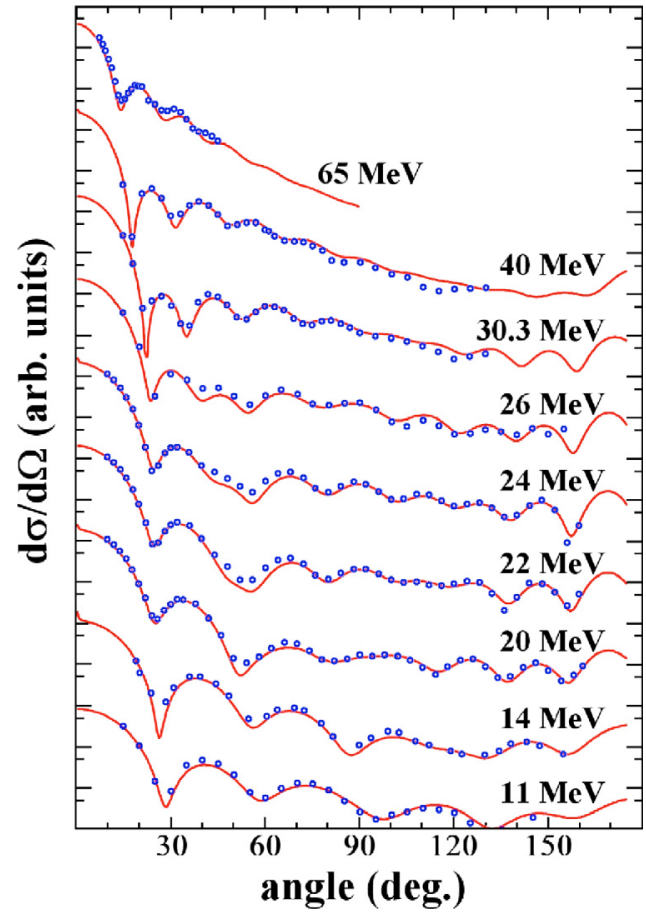

Fig. 5. Neutron elastic scattering off ${ }^{208} \mathrm{~Pb}$.

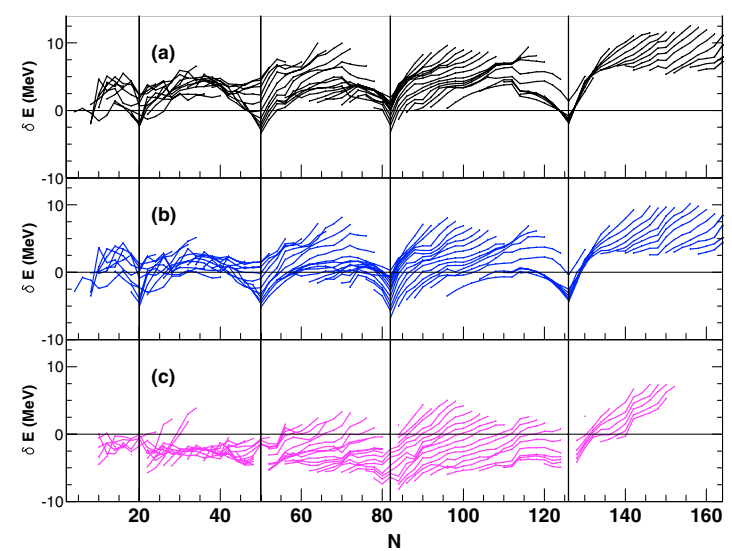

Fig. 6. Energy differences between theoretical and experimental masses of ref. [20] as functions of the neutron number for eveneven nuclei only. (a) Values contained in the database. (b) Predictions taking into account finite bases correction. (c) Predictions of (b) corrected for GCM + GOA dynamical effect.

Another extension has already been mentioned in the previous section. Indeed, we have seen that if the overall quality of the results contained in our database is quite reasonable, it is however clear that there are still significant disagreement which are known to stem from the fact that the axial hypothesis is not always good enough. Therefore, to improve the quality of the results, it is necessary to use the full quadrupole Generator Coordinate Method (GCM) with the Gaussian Overlap Approximation (GOA) to account for dynamical corrections [24]. Such time consuming calculations are presently underway and should be available in the near future. As can be observed on figures 6 and 7, the quality of the predictions is improved using this more complex method. 


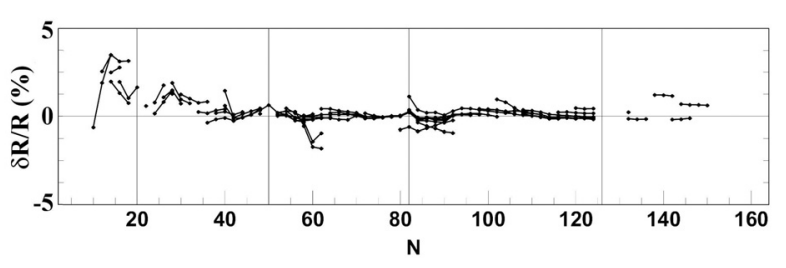

Fig. 7. Relative difference between theoretical $(\mathrm{HFB}+\mathrm{GCM}+\mathrm{GOA})$ and experimental charge radii as function of the neutron number. Experimental radii are taken from ref. [22].

On figure 6, we have plotted the same energy difference as in figure 3 for three cases for even-even nuclei only. The difference between pannel (a) and (b) is simply due to the fact that we account in pannel (b) for infinite basis corrections [21] whose effect is to reduce the mean deviation from $4.40 \mathrm{MeV}$ (a) down to $2.64 \mathrm{MeV}$ (b) by, on the average, translating down the theoretical binding energies of approximately 2-3 MeV. Pannel (c) illustrates the washing out of the arches initially present between the magic numbers when including dynamical corrections on top of the infinite basis ones. The mean deviation is then $2.94 \mathrm{MeV}$. This result is worse than that shown in pannel (b), but one must not forget that the D1S interaction overbounds a few nuclei. It is clear that if this overbinding effects were corrected, which would mean, to a first order approximation, shifting up the results shown in pannel (b), the washing out of the mid shell arches would improve the predicitions.

On figure 7, again, the improvement obtained including dynamical effects is clear. In this case it is due to the fact that the general trend of these effects is to increase the nucleus deformation and as a consequence increase its charge radius which is underestimated if axial symetry is imposed.

One last important point, for application, is the fact that apart from the improvement of the predicitons concerning the nuclear masses and radii, the discussed extension would also yield informations on low lying collective excited levels which are crucial for deformed optical model calculation as well as for level densities determination [25]. In this case, the excellent agreement [26] makes us believe that such extensions will be of great interest when prediction far from the experimentally accessed mass regions is required.

\section{Conclusions}

The nuclear AMEDEE structure datase has been put online at the web adress http://www-phynu.cea.fr/HFB-Gogny.htm. Its objective is to provide the scientific community with various data required when studying nuclei far from the valley of stability. Future extensions already let us expect significant improvements on the quality of the predicitions, and will also provide the nuclear reaction models with informations whose impact are known to be of great importance for reliable cross sections predictions.

\section{References}

1. J. Dechargé, D. Gogny, Phys. Rev. C 21, 1568 (1980).

2. J.F. Berger, M. Girod, D. Gogny, Comp. Phys. Comm. 63, 365 (1991).

3. J.P. Delaroche et al., Nucl. Phys. A 771, 103 (2006).

4. M. Kleban et al., Phys. Rev. C 65, 024309 (2002).

5. S. Hilaire, J.P. Delaroche, M. Girod, Eur. Phys. J. A 12, 169 (2001).

6. S. Peru, M. Girod, J.F. Berger, Eur. Phys. J. A 9, 35 (2000).

7. E. Bauge, J.P. Delaroche, M. Girod, Nucl. Phys. A 654, 829c (1999).

8. J. Libert, M. Girod, J.P. Delaroche, Phys. Rev. C 60, 054301 (1999).

9. X.H. Phan et al., Phys. Rev. C 38, 1173 (1988); Erratum Phys. Rev. C 39, 1645 (1989).

10. J.F. Berger, M. Girod, D. Gogny, Nucl. Phys. A 428, 23c (1984).

11. M. Girod et al., Phys. Rev. C 38, 1519 (1988).

12. M. Girod et al., Phys. Rev. Lett. 62, 2452 (1989).

13. P. Möller et al., At. Data Nucl. Data Tables 59, 185 (1995).

14. P. Möller, J.R. Nix, K.-L. Kratz, At. Data Nucl. Data Tables 66, 131 (1997)

15. Y. Aboussir et al., At. Data Nucl. Data Tables 61, 127 (1995).

16. S. Goriely, F. Tondeur, J.M. Pearson, At. Data Nucl. Data Tables 77, 311 (2001).

17. D. Lunney, J.M. Pearson, C. Thibault, Rev. Mod. Phys. 75, 1021 (2003).

18. M.V. Stoitsov et al., Phys. Rev. C 68, 054312 (2003).

19. G.A. Lalazissis, S. Raman, P. Ring, At. Data Nucl. Data Tables 71, 1 (1999).

20. A.H. Wapstra, G. Audi, C. Thibault, Nucl. Phys. A 729, 129 (2003).

21. M. Girod et al. (in preparation).

22. E.G. Nadjakov, K.P. Marinova, Y.P. Gangrsky, At. Data Nucl. Data Tables 56, 133 (1994); I. Angeli, At. Data Nucl. Data Tables 87, 185 (2004).

23. E. Bauge, J.P. Delaroche, M. Girod, Phys. Rev. C 63, 024607 (2001).

24. J. Libert, M. Girod, J.P. Delaroche, Phys. Rev. C 60, 054301 (1999).

25. S. Hilaire, S. Goriely, Nucl. Phys. A 779, 63 (2006) (these proceedings).

26. G.F. Bertsch et al. (submitted to Phys. Rev. Lett.). 